\title{
Prevalence of intermittent preventive treatment with sulphadoxine-pyrimethamine (IPTp-SP) use during pregnancy and other associated factors in Sekondi-Takoradi, Ghana
}

\author{
Verner N. Orish ${ }^{1}$, Onyekachi S. Onyeabor ${ }^{2}$, Johnson N. Boampong ${ }^{3}$, Richmond Afoakwah ${ }^{3}$, \\ Ekene Nwaefuna ${ }^{4}$, Samuel Acquah ${ }^{5}$, Adekunle O. Sanyaolu ${ }^{6,7}$, Nnaemeka C. Iriemenam ${ }^{6}$
}

1. Department of Internal Medicine, Effia-Nkwanta Regional Hospital Sekondi-Takoradi, Sekondi P. O. Box 229, Western Region, Ghana

2. The Satcher Health Leadership Institute, Department of Community Health and Preventive Medicine, Morehouse School of Medicine, Atlanta, Georgia, USA

3. Department of Human Biology, University of Cape Coast, Cape Coast, Central Region, Ghana

4. Biotechnology and Nuclear Agriculture Research Institute, Atomic Energy Commission, Accra, Ghana

5. Department of Medical Biochemistry, School of Medical Sciences, University of Cape Coast, Ghana

6. Department of Medical Microbiology and Parasitology, College of Medicine of the University of Lagos, Idi-araba, PMB 12003 Lagos, Nigeria

7. Saint James School of Medicine, Anguilla, BWI

\begin{abstract}
Background: Intermittent preventive treatment in pregnancy (IPTp) with sulphadoxine-pyrimethamine (SP) has been adopted as policy by most countries in sub-Saharan Africa. This cross-sectional study assessed the prevalence of IPTp-SP usage for prevention of malaria among pregnant women as well as evaluated factors associated with IPTp-SP use during pregnancy in Sekondi-Takoradi region of Ghana.

Methods: Pregnant women attending their antenatal-care with either clinical/ultrasound evidence of pregnancy were recruited. Venous blood was screened for malaria using RAPID response antibody kit and Giemsa staining. Haemoglobin estimations were done by cyanmethemoglobin method while Human Immunodeficiency Virus (HIV) screening was performed by the national diagnostic algorithm of two rapid antibody test and western blot confirmation.

Results: Of the 754 consented pregnant women interviewed in this study, 57.8\% had received IPTp-SP while $42.2 \%$ had not at their first contact with the study personnel. Furthermore, 18.6\% (81/436) of those that received IPTp-SP were malaria positive while $81.4 \%(355 / 436)$ were malaria negative. The results also indicated that $47.7 \%(51 / 107)$ of the pregnant women in their third trimester who were meant to have received at least two-doses of SP had received $\geq 2$ doses while $35.5 \%$ (38/107) had received 1 dose. In multivariable logistic regression analysis, pregnant women in their third trimester who received $\geq 2$ doses of SP showed decreased likelihoods of malaria (adjusted OR, 0.042; 95\% CI, 0.003-0.51; P = 0.013).

Conclusion: IPTp-SP usage among pregnant women in Sekondi-Takoradi reduces malaria and its use for malaria prevention should be strengthened with proper dosage completion and coverage.

Keywords: Malaria in pregnancy, IPTp-SP, anaemia, Ghana

DOI: http://dx.doi.org/10.4314/ahs.v15i4.6

Cite as: Orish VN, Onyeabor OS, Boampong JN, Afoakwah R, Nwaefuna E, Acquah S, et al. Prevalence of intermittent preventive treatment with sulphadoxine-pyrimethamine (IPTp-SP) use during pregnancy and other associated factors in Sekondi-Takoradi, Ghana. Afri Health Sci. 2015;15(4):1087-96. bttp:/ / dx.doi.org/10.4314/abs.v15i4.6
\end{abstract}

\author{
Corresponding author: \\ Verner N. Orish, \\ Department of Internal Medicine, \\ Effia-Nkwanta Regional Hospital \\ Sekondi-Takoradi, Sekondi \\ P. O. Box 229 Western Region, Ghana \\ Email: orishv@yahoo.com
}

\section{Introduction}

Malaria in pregnancy is an immense public health challenge, affecting about 50 million women per year, causing maternal, foetal and infant morbidity and mortality especially in sub-Saharan Africa ${ }^{1}$. Approximately 25 million pregnant women are at risk of Plasmodium falciparum infection which is the protozoan parasite that causes malaria in sub-Saharan Africa ${ }^{1}$. Among pregnant 
women in Ghana, malaria accounts for about 13.8\% of outpatient attendance and about $11 \%$ of maternal mortality. It is the leading cause of illness and death in the country impacting negatively on different socio-demographic groups ${ }^{2}$. The consequences of $P$. falciparum infection in pregnancy consist of maternal anaemia, low birth weight, preterm delivery, prenatal mortality, abortion, still birth, intrauterine growth retardation and even maternal mortality in some cases ${ }^{1,3-5}$. The severity of the adverse effects of malaria in pregnancy depends on gravidity and maternal age. However, primigravidae are at greater risk of malaria and its adverse effects than multigravidae and non-pregnant women from the same area $^{6}$.

World Health Organization (WHO) recommends the use of intermittent preventive treatment during pregnancy (IPTp) with sulphadoxine and pyrimethamine (SP), given at least twice at scheduled antenatal visits after quickening ${ }^{7}$.Ghana started this implementation in 2004 and the prevalence of placental malaria and maternal anaemia reduced substantially following the implementation of IPTp-SP ${ }^{8}$. The key goal of the global action plan is to cover $80 \%$ of the pregnant women in malaria endemic regions receiving no less than two doses of IPTp-SP in order to reduce the complications that arise from malaria in pregnancy ${ }^{9,10}$. The 2011 multiple indicator cluster survey from Ghana indicated progress on IPTp in Ghana although this has not been sustained $^{11}$. IPTp-SP administered to pregnant women in their second and third trimesters has been observed to reduce the risk of malaria ${ }^{12,13}$, placental malaria ${ }^{8}$, low birth weight and preterm births ${ }^{12-14}$, and maternal anae$\mathrm{mia}^{13,15}$. SP has a good safety profile ${ }^{16}$, and is cheap, cost effective and easy to administer through the already existing antenatal-care clinics (ANC) ${ }^{10,17}$.

Anaemia during pregnancy is a major global health problem, affecting nearly half of all pregnant women globally ${ }^{18}$. While the risk of anaemia in pregnancy increases with pregnancy progression ${ }^{18}$, the aetiology of anaemia in pregnancy is multifaceted ranging from undernourishment, iron deficiency, vitamins $A$ and $B_{12}$ deficiency, folate deficiency, sickle cell anaemia, thalassemia, Human Immunodeficiency Virus (HIV) infection, malaria and other parasites such as hookworm, Ascaris and Schistosoma ${ }^{19-23}$. However, in sub-Saharan Africa, malaria is a major contributor of anaemia ${ }^{22}$. Recently, studies showed that the influence of timing and fre- quency of $P$. falciparum malaria infection in late pregnancy were related to higher risk of maternal anaemia and low birth weight ${ }^{24,25}$. Although HIV infected pregnant women experience more malaria, dually infected pregnant women are more likely to have febrile illnesses ${ }^{26}$ and anaemia than women with either malaria or HIV single infection ${ }^{26,27}$.

While there are overwhelming support and evidence that IPTp-SP helps to reduce the risk of malaria when full doses are administered ${ }^{8,12-15,28-30}$, meeting the key goal coverage of $80 \%$ of the pregnant women receiving at least two doses of SP has been an uphill task ${ }^{31}$. We therefore conducted this cross-sectional study first to examine the prevalence of IPTp-SP usage for malaria prevention among pregnant women in Sekondi-Takoradi, secondly to evaluate the impact of IPTp-SP use on malaria prevalence among pregnant women in their third trimester meant to have received at least 2 doses of SP according to the WHO criteria ${ }^{7}$ and lastly, to study other factors associated with malaria among the pregnant women in the sub-region.

\section{Materials and methods \\ Study area and population}

This study was conducted at four hospitals in the Sekondi-Takoradi metropolis namely Effia Nkwanta Regional Hospital (the referral hospital in the region), Takoradi and Esikado Hospitals (Government hospitals) and Jemima Hospital (a private hospital). Details of the study population have been published elsewhere ${ }^{32}$. The hospitals are strategically located and were selected based on the size of attendance of pregnant women that come for their monthly ANC. Sekondi-Takoradi, the administrative capital of the western region of Ghana, is an industrial and commercial centre with a population of about 335,000 and a land area of 385 square kilometres. It is located about 242 kilometers to the West of Accra, the capital city and approximately 280 kilometers from the Côte d'Ivoire border. In this area, pregnant women (representing $4 \%$ of the population) are highly vulnerable to malaria infection ${ }^{2}$. The other highly vulnerable population are children under five years that constitute about $20 \%$ of the population.

\section{Recruitment of the pregnant women}

A cross-sectional study was carried out between March and October, 2010 at the earlier mentioned hospitals. Pregnant women attending their ANC visits with either 
clinical or ultrasound evidence of pregnancy were included in this study while those with severe bleeding were excluded from this study. Each facility was visited once every week on their routine antenatal days and all interviewed pregnant women were given adequate clinical attention and care by the respective hospitals. All consented pregnant women were recruited through the convenient sampling method, and were interviewed once (first contact with our study personnel) and their subsequent $\mathrm{ANC}$ visitations were not included in the data analysis. Interviews took place before their primary care consultations and pregnant women who received SP doses at their first contact with the study personnel were excluded from the data analysis. Written informed consents were obtained from all pregnant women that participated in this study. Ethical clearance was received from the Ghana Health Service Research Ethical Review Committee, Accra.

\section{Data collection}

A total of seven hundred and seventy six (776) pregnant women were interviewed out of which 754 gave their consent and were included in the data analysis. Information was obtained using a standardized questionnaire in English language interpreted in the patients' local language during the interviews. Two registered nurses who speak the same dialect translated the questionnaire information to the local language. Information on their socio-demographic characteristics, past and present obstetrics history, gravidae status, age, education, occupation, IPTp-SP use and other information were recorded. The numbers of SP doses taken by the pregnant women were documented on their ANC cards. History of fever and any other illness during the pregnancy were documented.

HIV screening for the pregnant women was performed and statuses of the women were obtained from the prevention of mother-to-child transmission (PMTC) clinic in the hospitals. Sero-statuses were determined by applying the national diagnostic algorithm of two rapid antibody tests and western blot confirmation while indeterminate cases were confirmed at the reference laboratory of the Regional Hospital. Five mls of venous blood was collected from the pregnant women by a trained laboratory technician from the median cubital vein. Blood samples were collected into an EDTA bottle and temporarily stored in an ice chest and were transported to a designated reference laboratory for same day analysis and storage.

Laboratory diagnosis of malaria was performed using fast RAPID response antibody kit (Premier Medical Corporation Ltd, Daman, India) and Giemsa staining. The brand of the RAPID response kit was specific for the detection of $P$. falciparum antigens. The presence of two lines in the test kit well indicated positive for $P$. falciparum malaria. For proper confirmation of malaria parasites, thick and thin films stained with Giemsa were prepared immediately upon blood collection on the same slide and examined microscopically using 100 power fields under oil immersion. The stained slides were read by 2 competent independent microscopists and where they had discordances, a third microscopist reassessed the slide. Haemoglobin estimation was performed using cyanmethemoglobin method ${ }^{33}$. Anaemia was defined based on WHO criteria haemoglobin levels of $<11 \mathrm{~g} / \mathrm{dL}^{34}$. Schistosoma haematobium was detected from wet mount preparation examination using microscopy of the concentrated sediments in urine. All laboratory personnel were blinded to the status of patients.

\section{Statistical analysis}

Socio-demographic characteristics of the pregnant women were based on Pearson's chi-squared test $(\chi 2)$ or exact chi-square for categorical variable and ANOVA for the comparison of the mean of the continuous variables. Age was dichotomized as adolescence and adults while the latter was used as the reference. Gravidity was categorized as primigravidae, secundigravidae and multigravidae using multigravidae as the reference in the logistic regression analysis. The number of SP doses received during pregnancy $(0,1$ and $\geq 2)$ were categorized and no SP doses (0) was used as the reference in the logistic regression models. Multivariable logistic regression analyses were used to assess the factors associated with malaria among the pregnant women controlling for age, gestation, gravidae, education, occupation, $S$. haematobium infection, fever, IPTp-SP use and HIV infection. Due to the multifactorial aetiology of anaemia in pregnancy, age, gravidae, occupation, education, IPTp-SP use, S. haematobium, fever, HIV and malaria infections were as well treated as covariates in the logistic regression models. Confounding was considered based on the results of univariable analysis, biological plausibility and, in addition, a $+/-10 \%$ change in odds ratio 
(OR) estimate. To assess the probable effect of IPTp$\mathrm{SP}$ use in the sub-region, pregnant women in their third trimester (who were expected to have received at least 2 doses of SP according to the WHO criteria ${ }^{7}$ ) were selected for further analysis using no SP doses as the indicator in the logistic regression model. All tests were two-tailed and $\mathrm{P}$ value of 0.05 or less was considered as statistically significant. The OR and $95 \%$ confidence interval (CI) were used to measure the strength of the associations. All statistical analyses were performed with IBM SPSS Statistics version 21.0 (IBM Corporation, Armonk, NY, USA).

\section{Results}

\section{Characteristics of the study participants}

Table 1 describes the general characteristics and obstetric history of the study population. Of the 754 consented pregnant women interviewed in this study, $57.8 \%$ had received IPTp-SP while $42.2 \%$ had not received IPTp-SP at their first contact with the study personnel. The mean ( \pm SD) age of the pregnant women was $26.17 \pm 5.5$ years. There were no statistically significant differences in the mean age of the pregnant women who received IPTp-SP $(26.3 \pm 5.6)$ and those who had not received IPTp-SP $(25.7 \pm 5.4)$ [Table 1].

\section{Table 1}

Characteristics and obstetric history of the pregnant women during their antenatal care visitation.

\begin{tabular}{|c|c|c|c|}
\hline $\begin{array}{l}\text { Socio-demographic } \\
\text { characteristics }\end{array}$ & $\begin{array}{l}\text { No IPTp-SP } \\
(\mathrm{n}=318)\end{array}$ & $\begin{array}{l}\text { IPTp-SP use } \\
(\mathrm{n}=436)\end{array}$ & $\begin{array}{l}\mathrm{P} \\
\text { value }\end{array}$ \\
\hline \multicolumn{4}{|l|}{ Age (years) } \\
\hline Mean $\pm \mathrm{SD}$ & $25.7 \pm 5.4$ & $26.3 \pm 5.6$ & 0.11 \\
\hline$\leq 19$ & 13.8 & 12.1 & \\
\hline $20-29$ & 62.6 & 59.4 & 0.13 \\
\hline $30-39$ & 23.3 & 26.4 & \\
\hline$>40$ & 0.3 & 2.1 & \\
\hline \multicolumn{4}{|l|}{ Education } \\
\hline None & 22.0 & 19.5 & \\
\hline Primary & 11.3 & 16.0 & 0.15 \\
\hline Secondary & 60.7 & 61.5 & \\
\hline Tertiary & 6.0 & 3.0 & \\
\hline \multicolumn{4}{|l|}{ Occupation } \\
\hline Civil service & 2.2 & 2.1 & \\
\hline Farmer/Trader/Caterer/Fishing & 94.2 & 96.6 & 0.27 \\
\hline Teacher/Student & 3.6 & 1.3 & \\
\hline \multicolumn{4}{|l|}{ Gravidae } \\
\hline Primigravidae & 62.9 & 55.4 & \\
\hline Secundigravidae & 28.3 & 35.5 & 0.11 \\
\hline Multigravidae & 8.8 & 9.1 & \\
\hline \multicolumn{4}{|l|}{ Trimesters } \\
\hline $1^{\text {st }}$ trimester & 38.9 & 0 & \\
\hline $2^{\text {nd }}$ trimester & 55.4 & 77.8 & $<0.001$ \\
\hline $3^{\text {rd }}$ trimester & 5.7 & 22.2 & \\
\hline \multicolumn{4}{|l|}{ Maternal anaemia } \\
\hline$<11 \mathrm{~g} / \mathrm{dL}$ & 31.8 & 36.2 & 0.22 \\
\hline$>11 \mathrm{~g} / \mathrm{dL}$ & 68.2 & 63.8 & \\
\hline \multicolumn{4}{|l|}{ Malaria status } \\
\hline Negative & 63.5 & 81.4 & $<0.001$ \\
\hline Positive & 36.5 & 18.6 & \\
\hline \multicolumn{4}{|l|}{ HIV status } \\
\hline Negative & 86.2 & 90.1 & 0.092 \\
\hline Positive & 13.8 & 9.9 & \\
\hline \multicolumn{4}{|l|}{ Febrile (fever) } \\
\hline Yes & 41.0 & 26.6 & $<0.001$ \\
\hline No & 59.0 & 73.4 & \\
\hline \multicolumn{4}{|l|}{ Admission } \\
\hline Yes & 1.6 & 2.5 & 0.37 \\
\hline No & 98.4 & 97.5 & \\
\hline \multicolumn{4}{|l|}{ Schistosoma haematobium } \\
\hline Negative & 85.5 & 88.9 & 0.17 \\
\hline Positive & 14.5 & 11.1 & \\
\hline \multicolumn{4}{|c|}{ Malaria co-infection with $S$. haematobium } \\
\hline Negative & 84.5 & 90.1 & 0.29 \\
\hline Positive & 15.5 & 9.9 & \\
\hline
\end{tabular}

NB: $\mathrm{N}=$ number of samples. $\mathrm{SD}=$ standard deviation. IPTp-SP $=$ intermittent preventive treatment with sulfadoxinepyrimethamine. Dataare percentages of the pregnant women.

$P$ values were based on Pearson chi-squared test or exact chi-square for categorical variables and ANOVA for the comparison of the mean of the continuous variables. 
There were also no statistically significant differences with education, occupation, gravidae, anaemia and HIV status of the pregnant women who received IPTp-SP and those who have not in univariable analysis. However, the proportion of pregnant women who received IPTp-SP and those who had not received IPTp-SP at their first contact with the study personnel differed with trimester, febrile and malaria status.

\section{IPTp-SP, SP doses and the prevalence of malaria} Thick and thin smears were used to detect malaria parasites from all the 754 recruited pregnant women. More than half of the pregnant women were malaria negative $73.9 \%$ (557) while $26.1 \%$ (197) were infected with malaria parasites. Among those that were infected with malaria parasites, $18.6 \%(81 / 436)$ received IPTp-SP while $36.5 \%(116 / 318)$ had not received IPTp-SP (Table 1). In addition, when stratified by the number of SP doses received, $20.7 \%$ of women who received one SP dose were malaria infected while $13.7 \%$ who received two doses of SP were malaria infected (Table 2). The results also indicated that the majority $86.3 \%(113 / 131)$ of the pregnant women who had received $\geq 2$ doses of SP were malaria negative (Table 2).

\section{Table 2}

Malaria statuses of the pregnant women by the number of SP doses.

\begin{tabular}{|c|c|c|c|c|}
\hline \multirow[t]{2}{*}{ Malaria status } & \multicolumn{3}{|c|}{ SP doses } & \multirow[b]{2}{*}{$P$ value } \\
\hline & $\begin{array}{l}0 \\
\mathrm{n}=318(\%)\end{array}$ & $\begin{array}{l}1 \\
\mathrm{n}=305(\%)\end{array}$ & $\begin{array}{l}\geq 2 \\
n=131(\%)\end{array}$ & \\
\hline Malaria negative & $202(63.5)$ & $242(79.3)$ & $113(86.3)$ & $<0.001$ \\
\hline Malaria positive & $116(36.5)$ & $63(20.7)$ & $18(13.7)$ & \\
\hline
\end{tabular}

$\mathrm{P}$ value derived from Pearson Chi-Square test. $\mathrm{n}=$ number of pregnant women. $\mathrm{SP}=$ sulphadoxine-pyrimethamine.

\section{IPTp-SP and haemoglobin levels}

Haemoglobin levels $(\mathrm{g} / \mathrm{dL})$ did not differ among those who received IPTp-SP and those who have not received. However, when those that received IPTp were selected for further analysis, pregnant women who received 3 doses of SP had higher median haemoglobin levels as compared with other doses though it did not reach statistical significance. No statistical significance was also observed with pairwise multiple comparisons of 0,1 and 2 doses with 3 doses.

\section{Risk factors associated with malaria among the pregnant women}

A separate model was run to assess the effect of factors associated with malaria infection among the recruited pregnant women. The possible confounding effects of age, adolescence, gravidae, gestational age, occupation, education and other variables were controlled us- ing multivariable logistic regression analysis. The data showed that pregnant women with tertiary education had reduced odds of malaria infection (adjusted OR, 0.39; 95\% CI, 0.15-0.99; P = 0.021) [Table 3]. However, HIV co-infection increased the odds of malaria in pregnancy by two-fold (adjusted OR, 2.37; $95 \% \mathrm{CI}$, 1.35-4.16; $\mathrm{P}=0.003)$. Similarly, women who were anaemic were two times more likely to have malaria when compared to women who were not anaemic (adjusted OR, 1.98; $95 \%$ CI, 1.28-3.06; P $=0.002$ ). In a separate model to explore the association between IPTp-SP use and malaria infection in the study population, 1 or $\geq 2$ SP doses was associated with preventing malaria in pregnancy. But, after regression assumption, confounding and interaction in multivariable logistic regression analysis, decreased odds of malaria was strongly associated with only $\geq 2$ doses of SP (adjusted OR, 0.41; 95\% CI, 0.19-0.88; $\mathrm{P}=0.023$ ) [Table 3]. 
Multivariable logistic regression analyses of factors associated with malaria among the pregnant women.

\begin{tabular}{|c|c|c|c|c|}
\hline Variables & $\mathrm{n}$ & Unadjusted OR (95\% CI) & *Adjusted OR (95\% CI) & $P$ value \\
\hline \multicolumn{5}{|l|}{ Age (years) } \\
\hline$\leq 19$ & 97 & $1.95(1.26-3.01)$ & $0.96(0.91-1.02)$ & 0.15 \\
\hline$\geq 20$ & 657 & 1 & 1 & \\
\hline \multicolumn{5}{|l|}{ Gravidae } \\
\hline Primigravidae & 441 & $0.72(0.43-1.21)$ & $0.54(0.24-1.24)$ & 0.14 \\
\hline Secundigravidae & 245 & $0.50(0.28-0.89)$ & $0.54(0.26-1.12)$ & 0.097 \\
\hline Multigravidae & 68 & 1 & 1 & \\
\hline \multicolumn{5}{|l|}{ Education } \\
\hline None & 155 & 1 & 1 & \\
\hline Primary & 106 & $0.98(0.58-1.66)$ & $1.34(0.67-2.67)$ & 0.41 \\
\hline Secondary & 461 & $0.76(0.51-1.13)$ & $0.90(0.52-1.54)$ & 0.69 \\
\hline Tertiary & 32 & $0.58(0.23-1.50)$ & $0.39(0.15-0.99)$ & 0.021 \\
\hline \multicolumn{5}{|c|}{ Schistosoma haematobium } \\
\hline Negative & 659 & 1 & 1 & \\
\hline Positive & 95 & $0.92(0.57-1.47)$ & $0.95(0.52-1.75)$ & 0.88 \\
\hline \multicolumn{5}{|l|}{ HIV } \\
\hline Negative & 667 & 1 & 1 & \\
\hline Positive & 87 & $4.31(2.73-6.79)$ & $2.37(1.35-4.16)$ & 0.003 \\
\hline \multicolumn{5}{|l|}{ Maternal anaemia } \\
\hline No & 495 & 1 & 1 & \\
\hline Yes & 259 & $2.05(1.48-2.82)$ & $1.98(1.28-3.06)$ & 0.002 \\
\hline \multicolumn{5}{|l|}{ IPTp-SP use } \\
\hline 0 & 318 & 1 & 1 & \\
\hline 1 & 305 & $0.45(0.32-0.65)$ & $0.69(0.42-1.11)$ & 0.13 \\
\hline$\geq 2$ & 131 & $0.28(0.16-0.48)$ & $0.41(0.19-0.88)$ & 0.023 \\
\hline
\end{tabular}

$\overline{N B}$ : OR = odds ratio. $\mathrm{CI}=$ confidence interval.

*Adjusted ORs are derived from multivariable binary logistic regression, controlling for age, adolescent, gestation, gravidae, education, occupation, febrile (fever), IPTp-SP use and HIV status. $\mathrm{P}<0.05$ was considered statistical significance.

\section{IPTp-SP use in third trimester}

To evaluate the impact of SP doses on malaria prevalence in the sub-region, only pregnant women in their third trimester, who were supposed to have received at least 2 doses of SP by the WHO recommendation, were selected for further analysis. The result indicated that $83.2 \%(89 / 107)$ of the pregnant women in their third trimester received SP. Out of these, $47.7 \%$
$(51 / 107)$ received $\geq 2$ doses of SP and $35.5 \%(38 / 107)$ received only 1 dose while $16.8 \%(18 / 107)$ received no SP doses [Table 4]. The data also indicated that $84.3 \%$ $(43 / 51)$ of those that received $\geq 2$ doses of SP were malaria negative while $15.7 \%(8 / 51)$ were malaria positive. In multivariable modelling, receipt of $\geq 2$ SP doses was statistically significantly associated with reduced risk of malaria infection (adjusted OR, 0.042; 95\% CI, 0.0030.51; P = 0.013) [Table 4]. 


\section{Table 4}

The effect of IPTp-SP use on the pregnant women during their third trimester.

\begin{tabular}{llllll}
\hline $\begin{array}{l}\text { SP doses } \\
(\mathrm{n}=107)\end{array}$ & \multicolumn{2}{l}{ Malaria status } & Unadjusted OR (95\% CI) & Adjusted OR (95\% CI) & P value \\
\cline { 2 - 4 } & Negative & Positive & & & \\
\hline & & & & 1 & \\
$0(18)$ & $11(61.1)$ & $7(38.9)$ & 1 & $0.11(0.14-0.83)$ & 0.032 \\
$1(38)$ & $30(78.9)$ & $8(21.1)$ & $0.42(0.12-1.43)$ & $0.042(0.003-0.51)$ & 0.013 \\
$\geq 2(51)$ & $43(84.3)$ & $8(15.7)$ & $0.29(0.087-0.98)$ & & \\
\hline
\end{tabular}

Adjusted ORs are derived from multivariable logistic regression analysis, controlling for age, gestation, gravidae, febrile, HIV and occupation.

\section{Discussion}

In this study, our results showed that pregnant women who received $\geq 2$ doses of SP during their third trimester had significantly decreased likelihoods of malaria. This observation is analogous with previous data from different malaria regions of sub-Saharan Africa ${ }^{8,12-14}$. The data also showed that the majority of the recruited pregnant women $(81.4 \%)$ who received IPTp-SP were malaria negative. However, $15.7 \%$ of the pregnant women in their third trimester that had received $\geq 2$ doses of SP were malaria positive. This observation could be as a result of possible re-infection or probably by the increase of SP drug resistance in pregnant women in Ghana since the adoption and implementation of IPTp-SP policy ${ }^{35}$. Furthermore, the number of doses given to pregnant women could be an important factor in IPTp-SP usage ${ }^{36}$ as there were significant differences in malaria prevalence with regard to the number of SP doses received by the pregnant women.

IPTp-SP is believed to be tolerable in pregnancy with no evidence of teratogenicity once given during the second and third trimesters ${ }^{16}$. However, despite the efficacy and cost effectiveness of IPTp-SP, SP drug resistance have been reported in Ghana ${ }^{35,37}$, as seen in most part of sub-Saharan Africa ${ }^{38}$ and debate over its safety and efficacy continues ${ }^{39-41}$. IPTp-SP had been shown to reduce the risk of anaemia especially in pregnant wom$\mathrm{en}^{15}$. In our cross-sectional study, we found no haemoglobin levels differences between those who received IPTp-SP and those who had not received IPTp-SP during the study period. This discrepancy could be as a result of study design differences. The earlier study utilised a smaller sample size (363) which may have had decreased precision when estimating unknown param- eters while our cross-sectional study utilised a higher sample size (754). However, the haemoglobin levels of pregnant women who received 3 doses of SP tended to be higher than those with 1-2 doses though it was not statistically significant.

The attainment of $80 \%$ coverage of pregnant women in malaria endemic region, like in Ghana, receiving at least two doses of IPTp-SP remains an uphill task. In a previous study, early ANC attendance, highly motivated personnel and compliance by pregnant women favoured the completion of IPTp ${ }^{42}$. But, earlier data from Uganda indicated that ANC visits do not actually ensure access to IPTp-SP in the presence of other barriers like stock-outs, long distance, irregular ANC visits or late ANC attendance ${ }^{43}$. In our study population, $47.7 \%$ of the pregnant women in their third trimester who were meant to have received $\geq 2$ doses of SP had completed the dosage. The reason for this low coverage with IPTp-SP could be related to late attendance to ANC, lack of completion of ANC visits and weak health services ${ }^{44}$. The lack or inconsistency in prenatal service attendance in some communities indicates that some pregnant women will not have access to IPTp-SP, some who have end up with less than the required doses before the delivery of the baby. This might still predispose them to anaemia and low birth weight of the baby. Three doses of IPTp-SP have been found to be more effective in malaria prevention than two doses as it halved the risk of placental malaria, low birth weight and preterm delivery ${ }^{14}$. Recently, a systematic review and meta-analysis suggested a new recommendation to provide at least 3 doses of SP especially during the second and third trimester ${ }^{45}$. It is imperative that measures like focused antenatal care and social mobilization be imple- 
mented to increase the proportion of pregnant women who take $\geq 2$ doses of SP in this sub-region. Furthermore, targeted education to women of child-bearing age needs more emphasis as studies have revealed the influential impact on malaria control ${ }^{46,47}$. This was evidenced in our study as pregnant women pregnant with tertiary education had reduced risk of malaria infection.

\section{Limitations}

There are few limitations to this study. First, none measurement of pregnancy outcomes like low birth weight and placental malaria, which are evidence of the association of malaria in pregnancy. Secondly, the use of insecticides treated mosquito bed nets was not included and this was not a randomized controlled clinical trial. This cross-sectional study allowed for a single encounter with the pregnant women and not all the pregnant women had an equal chance to receive IPTp. In addition, none measurement of other likelihood cause of anaemia also limited our analysis and none was followed till delivery. In view of the said, the interpretation of results should be based with reference to the observed. It would be ideal to conduct a controlled longitudinal study assessing the impact and effectiveness of IPTp use in the region and the potential factors involved in the selection of SP drug resistance.

\section{Conclusion}

This work further buttresses the importance of IPTpSP usage among pregnant women in preventing malaria and also shows the importance of dosage completion. Additional studies are recommended to assess the effectiveness of IPTp-SP use in a more detailed design that will measure low birth weight, placental malaria, the effects of insecticides treated mosquito bed nets and the drifts of SP drug resistance in the region. This is very essential in the wake of IPTp-SP drug resistance being reported by many workers.

\section{Presentation of results}

This result was presented in part at the 61st Annual ASTMH Meeting: November 11-15, 2012, Atlanta, Georgia, USA.

\section{Acknowledgments}

The authors wish to thank all patients for their involvement in this study. We are indebted to all the clinical and laboratory staff for their technical support. Our gratitude goes to the entire staff especially the maternity staff of Effia Nkwanta Regional Hospital, Esikado
Hospital, Takoradi Hospital and Jemima Hospital. We also thank the staff at Peace Laboratory.

\section{References}

1.Desai M, ter Kuile FO, Nosten F, McGready R, Asamoa K, Brabin B, Newman RD. Epidemiology and burden of malaria in pregnancy. Lancet Infect Dis 2007;7:93104.

2. PMI. Malaria Operational Plan for Ghana - Year Two (FY09). http://pmigov/countries/mops/fy09/ ghana_mop-fy09pdf (President's Malaria Initiative) 2009

3. Bodeau-Livinec F, Briand V, Berger J, Xiong X, Massougbodji A, Day KP, Cot M. Maternal anemia in Benin: prevalence, risk factors, and association with low birth weight. Am J Trop Med Hyg 2011;85:414-420.

4. Steketee RW, Nahlen BL, Parise ME, Menendez C. The burden of malaria in pregnancy in malaria-endemic areas. Am J Trop Med Hyg 2001;64:28-35.

5. van Geertruyden JP, Thomas F, Erhart A, D'Alessandro U. The contribution of malaria in pregnancy to perinatal mortality. Am J Trop Med Hyg 2004;71:35-40.

6. Brabin B. Malaria in pregnancy: current issues. Africa Health 1997;19:19-20.

7. WHO. A strategic framework for malaria prevention and control during pregnancy in the Africa region. Brazzaville, Congo: WHO Regional Office for Africa. AFR/MAL/04/01. 2004

8. Hommerich L, von Oertzen C, Bedu-Addo G, Holmberg V, Acquah PA, Eggelte TA, Bienzle U, Mockenhaupt FP. Decline of placental malaria in southern Ghana after the implementation of intermittent preventive treatment in pregnancy. Malar J 2007;6:144.

9. RBM. RBM Partnership. The global malaria action plan. Geneva: WHO. 2008

10. Oyibo WA, Agomo CO. Scaling up of intermittent preventive treatment of malaria in pregnancy using sulphadoxine-pyrimethamine: prospects and challenges. Matern Child Health J 2011;15:542-552.

11. Brieger B. Ghana: Progress on IPTp, but not yet sustained high coverage. http://wwwglobalhealthhuborg/2013/11/09/ghana-progress-on-iptp-but-notyet-sustained-high-coverage/ (Accessed June 6, 2014) 2013

12. Gies S, Coulibaly SO, Ouattara FT, D'Alessandro $\mathrm{U}$. Individual efficacy of intermittent preventive treatment with sulfadoxine-pyrimethamine in primi- and secundigravidae in rural Burkina Faso: impact on parasitaemia, anaemia and birth weight. Trop Med Int Health 2009;14:174-182. 
13. Aziken ME, Akubuo KK, Gharoro EP. Efficacy of intermittent preventive treatment with sulfadoxine-pyrimethamine on placental parasitemia in pregnant women in midwestern Nigeria. Int J Gynaecol Obstet 2011;112:30-33.

14. Maiga OM, Kayentao K, Traore BT, Djimde A, Traore B, Traore M, Ongoiba A, Doumtabe D, Doumbo S, Traore MS, Dara A, Guindo O, Karim DM, Coulibaly S, Bougoudogo F, Ter Kuile FO, Danis M, Doumbo OK. Superiority of 3 over 2 doses of intermittent preventive treatment with sulfadoxine-pyrimethamine for the prevention of malaria during pregnancy in mali: a randomized controlled trial. Clin Infect Dis 2011;53:215223.

15. Wilson NO, Ceesay FK, Obed SA, Adjei AA, Gyasi RK, Rodney P, Ndjakani Y, Anderson WA, Lucchi NW, Stiles JK. Intermittent preventive treatment with sulfadoxine-pyrimethamine against malaria and anemia in pregnant women. Am J Trop Med Hyg 2011;85:12-21. 16. Peters PJ, Thigpen MC, Parise ME, Newman RD. Safety and toxicity of sulfadoxine/pyrimethamine: implications for malaria prevention in pregnancy using intermittent preventive treatment. Drug Saf 2007;30:481501.

17. Goodman CA, Coleman PG, Mills AJ. The cost-effectiveness of antenatal malaria prevention in sub-Saharan Africa. Am J Trop Med Hyg 2001;64:45-56.

18. Lee AI, Okam MM. Anemia in pregnancy. Hematol Oncol Clin North Am 2011;25:241-259, vii.

19. WHO. Worldwide prevalence of anaemia 1993-2005. WHO Global Database on Anaemia http://whqlibdocwhoint/publications/2008/9789241596657_engpdf 2008

20. van den Broek N. Anaemia in pregnancy in developing countries. BrJ Obstet Gynaecol 1998;105:385-390.

21. Verhoeff FH, Brabin BJ, Chimsuku L, Kazembe P, Broadhead RL. An analysis of the determinants of anaemia in pregnant women in rural Malawi--a basis for action. Ann Trop Med Parasitol 1999;93:119-133.

22. Savage EJ, Msyamboza K, Gies S, D'Alessandro U, Brabin BJ. Maternal anaemia as an indicator for monitoring malaria control in pregnancy in sub-Saharan Africa. BJOG 2007;114:1222-1231.

23. Ghosh K, Ghosh K. Pathogenesis of anemia in malaria: a concise review. Parasitol Res 2007;101:1463-1469. 24. Kalilani L, Mofolo I, Chaponda M, Rogerson SJ, Meshnick SR. The effect of timing and frequency of Plasmodium falciparum infection during pregnancy on the risk of low birth weight and maternal anemia. Trans R Soc Trop Med Hyg 2010;104:416-422.
25. Huynh BT, Fievet N, Gbaguidi G, Dechavanne S, Borgella S, Guezo-Mevo B, Massougbodji A, Ndam NT, Deloron P, Cot M. Influence of the timing of malaria infection during pregnancy on birth weight and on maternal anemia in Benin. Am J Trop Med Hyg 2011;85:214-220.

26. ter Kuile FO, Parise ME, Verhoeff FH, Udhayakumar V, Newman RD, van Eijk AM, Rogerson SJ, Steketee RW. The burden of co-infection with human immunodeficiency virus type 1 and malaria in pregnant women in sub-saharan Africa. Am J Trop Med Hyg 2004;71:41-54.

27. Orish VN, Onyeabor OS, Boampong JN, Acquah $\mathrm{S}$, Sanyaolu AO, Iriemenam NC. The effects of malaria and HIV co-infection on hemoglobin levels among pregnant women in Sekondi-Takoradi, Ghana. Int J Gynaecol Obstet 2013;120:236-239.

28. Falade CO, Yusuf BO, Fadero FF, Mokuolu OA, Hamer DH, Salako LA. Intermittent preventive treatment with sulphadoxine-pyrimethamine is effective in preventing maternal and placental malaria in Ibadan, south-western Nigeria. Malar J 2007;6:88.

29. Challis K, Osman NB, Cotiro M, Nordahl G, Dgedge M, Bergstrom S. Impact of a double dose of sulphadoxine-pyrimethamine to reduce prevalence of pregnancy malaria in southern Mozambique. Trop Med Int Health 2004;9:1066-1073.

30. Gutman J, Mwandama D, Wiegand RE, Ali D, Mathanga DP, Skarbinski J. Effectiveness of intermittent preventive treatment with sulfadoxine-pyrimethamine during pregnancy on maternal and birth outcomes in Machinga district, Malawi. J Infect Dis 2013; 208:907-916.

31. d'Almeida TC, Agboton-Zoumenou MA, Garcia A, Massougbodji A, Briand V, Imorou Y, Cottrell G. Field evaluation of the intermittent preventive treatment of malaria during pregnancy (IPTP) in Benin: evolution of the coverage rate since its implementation. Parasit Vectors 2011;4:108.

32. Orish VN, Onyeabor OS, Boampong JN, Aforakwah R, Nwaefuna E, Iriemenam NC. Adolescent pregnancy and the risk of Plasmodium falciparum malaria and anaemia-a pilot study from Sekondi-Takoradi metropolis, Ghana. Acta Trop 2012;123:244-248.

33. Bhaskaram P, Balakrishna N, Radhakrishna KV, Krishnaswamy K. Validation of hemoglobin estimation using Hemocue. Indian J Pediatr 2003;70:25-28.

34. WHO. Preventing and controlling iron deficiency anaemia through primary health care, WHO Geneva. http://wwwwhoint/nutrition/publications/micronu- 
trients/anaemia_iron_deficiency/9241542497pdf (Accessed January 4, 2013) 1989

35. Mockenhaupt FP, Bedu-Addo G, Eggelte TA, Hommerich L, Holmberg V, von Oertzen C, Bienzle $\mathrm{U}$. Rapid increase in the prevalence of sulfadoxine-pyrimethamine resistance among Plasmodium falciparum isolated from pregnant women in Ghana. J Infect Dis 2008;198:1545-1549.

36. Gill CJ, Macleod WB, Mwanakasale V, Chalwe V, Mwananyanda L, Champo D, Mukwamataba D, Chilengi R, Thea DM, Hamer DH. Inferiority of single-dose sulfadoxine-pyrimethamine intermittent preventive therapy for malaria during pregnancy among HIV-positive Zambian women. J Infect Dis 2007;196:1577-1584. 37. Mockenhaupt FP, Teun Bousema J, Eggelte TA, Schreiber J, Ehrhardt S, Wassilew N, Otchwemah RN, Sauerwein RW, Bienzle U. Plasmodium falciparum dhfr but not dhps mutations associated with sulphadoxine-pyrimethamine treatment failure and gametocyte carriage in northern Ghana. Trop Med Int Health 2005;10:901-908.

38. Sridaran S, McClintock SK, Syphard LM, Herman KM, Barnwell JW, Udhayakumar V. Anti-folate drug resistance in Africa: meta-analysis of reported dihydrofolate reductase (dhfr) and dihydropteroate synthase (dhps) mutant genotype frequencies in African Plasmodium falciparum parasite populations. Malar J 2010;9:247.

39. Gutman J. Editorial commentary: intermittent preventive treatment in pregnancy with sulfadoxine-pyrimethamine: the controversy continues. Clin Infect Dis 2012;55:1103-1105.

40. Harrington W, McGready R, Muehlenbachs A, Fried M, Nosten F, Duffy P. Intermittent preventive treatment in pregnancy with sulfadoxine-pyrimethamine: the times they are a-changin'. Clin Infect Dis 2012;55:1025-1026.
41. McGready R, White NJ, Nosten F. Parasitological efficacy of antimalarials in the treatment and prevention of falciparum malaria in pregnancy 1998 to 2009: a systematic review. BJOG 2011;118:123-135.

42. Olliaro PL, Delenne H, Cisse M, Badiane M, Olliaro A, Vaillant M, Brasseur P. Implementation of intermittent preventive treatment in pregnancy with sulphadoxine/pyrimethamine (IPTp-SP) at a district health centre in rural Senegal. Malar J 2008;7:234.

43. Ndyomugyenyi R, Katamanywa J. Intermittent preventive treatment of malaria in pregnancy (IPTp): do frequent antenatal care visits ensure access and compliance to IPTp in Ugandan rural communities? Trans $R$ Soc Trop Med Hyg 2010;104:536-540.

44. Osei Tutu E, Lawson B, Browne E. The effectiveness and perception of the use of sulphadoxine-pyrimethamine in intermittent preventive treatment of malaria in pregnancy programme in Offinso District of Ashanti Region, Ghana. Malar J 2011;10:385.

45. Kayentao K, Garner P, van Eijk AM, Naidoo I, Roper C, Mulokozi A, MacArthur JR, Luntamo M, Ashorn P, Doumbo OK, ter Kuile FO. Intermittent preventive therapy for malaria during pregnancy using 2 vs 3 or more doses of sulfadoxine-pyrimethamine and risk of low birth weight in Africa: systematic review and meta-analysis. JAMA 2013;309:594-604.

46. Iriemenam NC, Dosunmu AO, Oyibo WA, Fagbenro-Beyioku AF. Knowledge, attitude, perception of malaria and evaluation of malaria parasitaemia among pregnant women attending antenatal care clinic in metropolitan Lagos, Nigeria. J Vector Borne Dis 2011;48:1217.

47. Takem EN, Achidi EA, Ndumbe PM. Use of intermittent preventive treatment for malaria by pregnant women in Buea, Cameroon. Acta Trop 2009;112:54-58. 